\title{
A Web-based Electronic Health Record System for National Surveillance
}

\author{
Jake R. Marcus* \\ Practice Fusion, San Francisco, CA, USA
}

\section{Objective}

This showcase aims to demonstrate the viability of Practice Fusion's web-based electronic health record system for national surveillance. Practice Fusion also wishes to provide aggregate data to public health departments for surveillance for free. This showcase also hopes to engage those potential partners around uses of the company's research database.

\section{Introduction}

Practice Fusion is a web-based electronic health record system with over 150,000 medical professional users treating over 50 million patients. The company focuses on small, ambulatory practices and is predominately comprised of practices in the field of primary care. The user base makes it an ideal system for public health surveillance. The Research Division has undertaken pilot projects to demonstrate the viability of using the data for surveillance for acute diseases, like influenza-like illness, chronic diseases, like diabetes, and risk factors, like hypertension.

\section{Methods}

Surveillance systems based on electronic health records have clear advantages over case based reporting, but the majority of those systems are limited to the small geographical area affiliated with the hospital or health plan associated with the project. Practice Fusion has coverage in all 50 states and runs on a single, multi-tenant database making comparisons across those states and the localities within them immediately feasible. The company wishes to engage the organizations represented at ISDS in order to advance public health surveillance using the research database. It is very difficult to obtain electronic health record data currently, but with the appropriate data use agreement Practice Fusion believes that it is a moral imperative to use its aggregate data for surveillance. The Research Division has developed methods for the surveillance of influenza-like illness with its system and comparisons to the $\mathrm{CDC}$ have proven its viability.

\section{Conclusions}

By comparing trends and levels of influenza-like illness generated from Practice Fusion's research database to Google Flu Trends and the gold standard estimates produced by the CDC, web-based electronic health record systems have proved to be a viable foundation for syndromic surveillance. The implementation of the system also shows that case definitions for surveillance need not be overly simplistic if they do not require cases to be submitted from physicians, but rather can be programmed to be identified through automated algorithms.

\section{Keywords}

public health surveillance; electronic health record; Practice Fusion

\section{Acknowledgments}

The data team at Practice Fusion

\section{References}

www.practicefusion.com

research.practicefusion.com

*Jake R. Marcus

E-mail: jmarcus@practicefusion.com 